index comunicación | no 11(2) 2021 | Páginas 257-281

E-ISSN: 2174-1859 | ISSN: 2444-3239 | Depósito Legal: M-19965-2015

Recibido el 17_01_2021 | Aceptado el 24_05_2021 | Publicado el 15_07_2021

\title{
UN MODELO DE ANÁLISIS POLÍTICO COMPARADO PARA VIDEOJUEGOS DE SIMULACIÓN DE GOBIERNO. ESTUDIO DE CASO CON 'FROSTPUNK', 'TROPICO IV' Y'CIVILIZATION V'
}

\author{
A COMPARATIVE POLITICAL ANALYSIS MODEL \\ FOR GOVERNMENT SIMULATION VIDEOGAMES. \\ CASE STUDY OF 'FROSTPUNK', 'TROPICO IV' \\ AND 'CIVILIZATION V'
}

https://doi.org/10.33732/ixc/11/02Unmode

Carlos Moreno Azqueta Universidad de Castilla-La Mancha carlosmorenoazqueta@gmail.com https://orcid.org/0000-0001-5771-3069 
Resumen: En este artículo se presenta un modelo de análisis comparativo a gran escala para videojuegos en los que el jugador gestiona el desarrollo de una comunidad política, que llamaremos «simuladores de gobierno». El modelo, construido sobre literatura de ciencia política y otros modelos analíticos de videojuegos, usa un conjunto de variables operacionalizadas para sintetizar los componentes del sistema político representados en los juegos, así como la estructura de objetivos y elementos que orientan la acción del jugador, para finalmente compararlos con un conjunto de variables independientes. Pondremos a prueba el modelo aplicándolo a tres famosos simuladores de gobierno que han recibido notable atención académica (Civilization V, Tropico IV y Frostpunk), para finalmente discutir sus ventajas y límites.

Palabras clave: videojuegos; simuladores de gobierno; modelo de análisis; Civilization; Tropico; Frostpunk.

\begin{abstract}
In this paper we present a large-scale comparative analysis model for videogames in which the player manages the development of a political community, which we will call «government simulators». The model, built upon political-science literature and other analytical models for videogames, uses a set of operationalized variables to synthesize the components of the political system represented in games, as well as the structure of objectives and other elements that guide the player's action, to finally compare them with a set of independent variables. The model will be tested by applying it to three famous government simulators that have received notable academic attention (Civilization V, Tropico IV and Frostpunk), to finally discuss its advantages and limitations.
\end{abstract}

Keywords: Videogames; Government Simulator; Analytical Models; Civilization; Tropico; Frostpunk. 


\section{Introducción, justificación y marco teórico}

\subsection{Introducción}

La creciente importancia social y cultural de los videojuegos ha puesto sobre la mesa su capacidad para construir ideas y representaciones sobre la política y la sociedad, y ha abierto un campo disciplinar que se ocupa de analizar críticamente sus características ideológicas implícitas.

Un tipo particular de videojuegos, en los cuales el jugador gestiona y dirige el desarrollo de una comunidad política, y que aquí llamaremos "simuladores de gobierno», ha recibido especial atención académica (Dolkemeyer, 2020; McNeil, 2016; Planells, 2015a; Poblocki, 2002; Shields, 2009, entre otros), probablemente por sus potenciales efectos performativos en la socialización política de los jugadores. En este trabajo se propone un modelo de análisis para el estudio y comparación de simuladores de gobierno. La principal ventaja del modelo es que ofrece una operacionalización cuantitativa de sus variables que permite tratar muestras importantes de juegos de forma estandarizada. Nuestros objetivos pueden presentarse así:

- Objetivo general:

- Desarrollar un modelo de análisis político comparativo para videojuegos de simulación de gobierno.

- Objetivos específicos:

- Discutir de forma crítica la pertinencia, ventajas y límites de las variables presentes en el modelo.

- Aplicar el modelo a tres videojuegos de simulación de gobierno.

- Comentar las intersecciones entre ciencia política y de la administración y estudios sobre videojuegos a través del modelo.

En las próximas páginas se desarrollará una definición más precisa del concepto de "simuladores de gobierno», para luego exponer una breve revisión teórica de otros modelos de análisis de videojuegos y de los estudios de caso realizados en simuladores de gobierno. A continuación, se presentará la metodología del modelo, discutiendo las variables una a una, señalando la forma en la que interconectan aspectos videolúdicos y relativos a la teoría política, y poniéndolo a prueba a través de su aplicación a tres simuladores de gobierno que han sido objeto habitual de atención académica: Civilization $V$ (Firaxis Games, 2010; en adelante Civilization), Tropico IV (Haeminont Games, 2011; en adelante Tropico) y Frostpunk (11 bit studios, 2018). Por último, se expondrá una revisión de los potenciales límites a los que se enfrenta. 


\subsection{Definición del objeto de estudio}

Aunque el término simulador de gobierno no se emplea aún en la industria o los game studies, tiene especial interés analítico por su conexión con las ciencias políticas y de la administración. Aquí será definido como un videojuego en el que el jugador toma el control de una comunidad política y gestiona su desarrollo. Al mismo tiempo, se definirá comunidad política como un grupo de personas que, viviendo en el mismo territorio, se organizan políticamente de forma autónoma (aunque no necesariamente democrática), estableciendo y aceptando normas e instituciones que regulan la vida en común. Estas comunidades pueden ser ciudades, grupos de refugiados, imperios o colonias en planetas desconocidos, entre otras.

Normalmente, los simuladores de gobierno entran dentro de la etiqueta de «juegos de estrategia», pero dicha etiqueta es poco operativa (Dor, 2018). La gran mayoría de ellos se aglutinan en tres subgéneros:

1. Los juegos de construcción de ciudades, con comunidades políticas reducidas y un énfasis en la planificación urbana. Tanto Tropico como Frostpunk son juegos de construcción de ciudades, aunque probablemente el más famoso sea Sim City (Maxis, 1989).

2. Los llamados $4 \mathrm{X}$, cuyas siglas vienen a significar eXplore, eXpand, eXploit $y$ eXterminate. En estos juegos el jugador construye un gran imperio que debe imponerse, habitualmente de forma militar, al de sus rivales. Civilization forma parte de este grupo.

3. Los «simuladores políticos». Este es el subgénero más minoritario, y su nombre no es especialmente explicativo, además de aplicarse ocasionalmente a juegos de otros géneros. Se trata de simuladores de gobierno ambientados en estados-nación modernos, como Democracy 3 (Positech Games, 2013), donde el énfasis del juego se encuentra en la adopción de políticas públicas, la gestión económica y presentarse a elecciones para mantenerse en el poder.

La elección de los simuladores de gobierno como objeto de estudio tiene que ver con su especial interés desde el punto de vista de los estudios de comunicación y de la teoría política. En primer lugar, porque presentan «mundos posibles» (Planells, 2015b) con complejos sistemas políticos de los cuales los jugadores pueden aprender (Pérez et al., 2008) y, como todo videojuego, reglas de funcionamiento que transmiten valoraciones sobre lo correcto y lo incorrecto (Bogost, 2007). En segundo lugar, porque los jugadores participan de forma directa en la gestión de esa comunidad política, pudiendo en algunos casos determinar su desarrollo ideológico. Ambos aspectos 
combinados llevan consigo un potencial de estimulación de la imaginación política de los jugadores que justifica nuestro interés en la cuestión.

\subsection{Marco teórico}

Uno de los primeros desafíos a los que se han enfrentado los game studies ha sido metodológico. Los videojuegos son textos extraordinariamente complejos, tanto por cómo incorporan elementos de muchos medios distintos (lo que en ocasiones se ha llamado «remediación») como por su componente interactivo. De las diversas ramas de análisis que han surgido (contextos de producción, comunidades de jugadores...) aquí hablaremos solo de los análisis de contenido de los juegos. Pero dentro de esa categoría han surgido múltiples modelos de análisis. Hasta donde llega el conocimiento del autor, no han sido propuestos modelos de análisis comparativos y cuantitativos para simuladores de gobierno o un género similar. Sin embargo, sí existen muchos modelos de análisis, normalmente cualitativos, de los que bebe esta propuesta.

Óliver Pérez Latorre (2010) presenta una propuesta particularmente completa que incorpora una triple perspectiva al estudio del videojuego: ludológico-sistémica, narrativa y semiótica. La incorporación de la semiótica es posiblemente el aspecto más original, que el autor desarrolla posteriormente en el estudio de caso de The Last of us (Latorre, Oliva y Besalú, 2016). Tanto Latorre (2010) como Navarro (2016) presentan en sus modelos extensas «guías» de análisis que dan cuenta de todos los aspectos que pueden ser estudiados y ofrecen una estructura de los componentes de un juego que ayudan a una lectura completa, de forma similar al Game Ontology Project, una suerte de wikipedia de conceptos relacionados con los videojuegos. Navarro (2016), por su parte, propone en su modelo una forma particular de entender la agencia del jugador como «libertad dirigida», condicionada por los elementos de diseño.

Planells (2015b), por otra parte, presenta un modelo centrado en lo que llama «mundos posibles». Emplea la filosofía de los mundos posibles y la lógica modal para desarrollar un modelo que estudia las características de los mundos videolúdicos y su dinamismo. El énfasis en la diversidad de posibilidades resalta de esa forma el potencial de los videojuegos para estimular la imaginación política de los jugadores.

Por otra parte, los estudios de caso sobre simuladores de gobierno concretos (y en particular los tres que emplearemos para el análisis) han hecho uso de modelos de estudio propios al tiempo que señalaban las interconexiones entre ciencia política y simuladores de gobierno. Shields (2009), por ejemplo, emplea un análisis basado en el «pentágono dramatúrgico» de Kenneth Burke (1945), un modelo que divide los textos o 
hechos estudiados en cinco elementos de un drama: el acto, la escena, el agente, la agencia y el propósito. Por su parte, Planells (2015a) aplica su modelo de mundos posibles a los mundos ideales de las distintas facciones de Tropico IV para mostrar la forma en que el juego representa la teoría del pluralismo político. Con respecto a Frostpunk, Chauriye (2019) emplea un marco de análisis de videojuegos en general llamado MDA, siglas que representan las tres patas del sistema: «Mechanics, Dynamics \& Aesthetics» (Robin et al., 2004). Dolkemeyer (2020) emplea a Haraway y sus conceptos de «response-ability» y Sympoiesis, discutiendo el papel del jugador-gobernante en la comunidad política. Con respecto a la saga Civilization, esta ha sido analizada desde la perspectiva decolonial en diversos trabajos (Douglas, 2002; Ford, 2016), o la noción foucaultiana de biopolítica (Poblocki, 2002). Por último, la propuesta más cercana al modelo que aquí proponemos podría encontrarse en Nicolás de Zamaroczy (2016), que discute varias asunciones erróneas con respecto a las relaciones internacionales presentes en videojuegos de estrategia populares, como el «control perfecto» o la «otredad radical». Estas asunciones podrían convertirse en variables de análisis comparativo similares a las que planteamos, aunque Zamaroczy no busca operacionalizarlas ni ofrecer un análisis completo.

Más allá de la ciencia política, otros trabajos han señalado conexiones entre videojuegos y ciencias de la administración. Exmeyer y Boden (2020), por ejemplo, estudian las representaciones de la administración pública en Papers, Please, en una tensión entre la rigidez impersonal y la discrecionalidad y desobediencia sancionada pero central en el juego. Los autores invitan a pensar la cuestión tanto desde la perspectiva de la representación en la cultura popular como de la experiencia lúdico-educativa que permite reflexionar sobre las propias dinámicas de la burocracia pública, una aproximación que compartimos: en muchos aspectos los simuladores de gobierno están relacionados con la planificación eficiente de políticas públicas, por lo que podemos pensarlos como artefactos culturales que ofrecen representaciones y planteamientos ideológicos, pero también como instrumentos prácticos de formación administradores públicos. Similar reflexión puede plantearse con las interconexiones políticas: estos juegos no solo presentan representaciones, sino que son objetos interactivos, formativos y experimentales, que pueden funcionar como "escuelas de ciudadanía», si bien en un sentido más abstracto.

Así pues, existe una importante variabilidad teórica y metodológica, en particular en los estudios de caso. Sin embargo, tanto estos como los modelos más generales raramente se expresan de forma cuantitativa, y presentan 
dificultades a la hora de estandarizar y comparar los resultados. No es raro en ese sentido que existan discrepancias interpretativas entre los autores, que surgen a partir de puntos de vista y metodologías analíticas distintas (aunque los acuerdos generales son más comunes). Los estudios comparativos, a su vez, se limitan a un pequeño número de juegos y a aspectos muy concretos, como los árboles de tecnología de los juegos (Ghys, 2002; Zamaroczy, 2016). El modelo que aquí se presenta busca abordar modestamente ese déficit de comparabilidad y capacidad de estandarización de la literatura actual sobre simuladores de gobierno, al tiempo que se alimenta de sus aportaciones.

\section{Introducción al modelo y los juegos}

\subsection{Introducción al modelo}

Esta propuesta de modelo de análisis se alimenta de la literatura anteriormente presentada (modelos generales de análisis, estudios de caso y literatura sobre ciencia política y de la administración). Parte de una perspectiva ludológica-sistémica incorporando elementos semióticos (siguiendo a Latorre, 2010), pero excluye aspectos narrativos para simplificar la aplicación. Al estar pensado específicamente para simuladores de gobierno, es más concreto que los modelos de análisis general de videojuegos, pero también más general que los estudios de caso. También es de los pocos modelos con expresión cuantitativa. En términos resumidos, las principales utilidades del modelo son las siguientes:

- Permite estudiar y presentar las características fundamentales de una muestra de videojuegos a gran escala.

- Facilita comparar los videojuegos estudiados entre sí gracias a la estandarización cuantitativa de sus variables.

- Permite estudiar (con una muestra significativa) las correlaciones entre variables independientes (como el género, la ambientación o el año de surgimiento del juego) y variables dependientes (relacionadas con las características del mundo videolúdico).

El modelo se compone de 18 variables, cada una de ellas con diversas opciones y un valor numérico asignado. 10 de ellas son variables de escala, facilitando el empleo de técnicas estadísticas como medias y medianas. Algunas de las variables incorporarán en la explicación "sub-indicadores», factores que deben servir de guía para determinar el valor que le corresponde a un juego en determinada variable. 
Las variables se organizan en cuatro grupos: sistema político, diseño de la acción del jugador, marcas enunciativas y variables independientes. De forma provisional, llamaremos a este modelo «modelo estandarizado de análisis político de videojuegos de simulación de gobierno», a la espera de un título más atractivo.

A continuación, se realizará un breve resumen de los juegos a los que vamos a aplicar el análisis, para después presentar cada una de las variables del modelo, con explicaciones relativas a su inclusión y funcionamiento y una breve discusión sobre los resultados al ser aplicadas a los tres juegos. Estos resultados no deben tomarse como resultados generales del modelo (que está pensado para una muestra mucho mayor de juegos), sino como ejemplos del tipo de conclusiones a las cuales podría llegarse, y de las dificultades que pueden presentarse.

\subsection{Juegos que van a ser analizados}

Las primeras entregas de la saga Civilization se remontan a los años 90, siendo de los primeros juegos del género 4X. En este videojuego encarnamos la figura de gobernante eterno de una civilización desde sus inicios, en el año 4000 a.C., hasta potencialmente el futuro. A partir de nuestro primer colono desarrollaremos nuestra civilización, investigaremos tecnologías y formas de organización social, e interactuaremos con otras comunidades. Todo ello en busca de alguna de las victorias posibles que el juego ofrece, que en Civilization $V$ son: científica (construyendo una nave para colonizar otro planeta), por dominación (conquistando las capitales de las demás civilizaciones), cultural (generando turismo hasta que otras civilizaciones adopten tu cultura), diplomática (siendo elegido líder mundial en el congreso de las naciones, lo que habitualmente incluye una cantidad inédita de sobornos y es por tanto considerada una victoria económica) o por puntuación (llegando al año 2050 con más puntos que nadie). En este trabajo se emplea la versión de Civilization $V$ (Firaxis Games, 2010) con todas las expansiones.

La saga Tropico, por su parte, fue lanzada en 2001. Es un juego de tono irónico, un constructor de ciudades en el que encarnamos la figura de presidente dudosamente democrático de una ficticia isla caribeña en el contexto de la Guerra Fría. El jugador debe desarrollar la economía y política de la isla y mantener a sus ciudadanos felices para lograr su aprobación y asegurarse su victoria electoral, que dependerá de la buena imagen que tenga entre las diversas facciones políticas: capitalistas, comunistas, religiosos, intelectuales, ecologistas y lealistas. También puede recurrir a métodos un tanto más heterodoxos, como el soborno, encarcelamiento o asesinato de líderes de facciones rivales, o el fraude electoral. Tales actos, sin embargo, llevarán a que algunos ciudadanos abandonen la vida civil y se organicen en una guerrilla contra el presidente. Todo esto se produce, por otra parte, en un 
tono paródico de fuerte cinismo político. Se empleará aquí la versión básica, sin expansiones, de Tropico IV (Haemintont Games, 2011).

Frostpunk es el juego más moderno de los seleccionados, surgido en 2018. En el contexto de un desastre climático de origen desconocido que ha llevado a la tierra a una glaciación, el jugador debe dirigir a un grupo de refugiados que están creando una nueva colonia (¿tal vez la última de la humanidad?) en torno a un enorme generador. Se trata de un constructor de ciudades de supervivencia, que destaca por un tono extremadamente serio, busca construir una sistemática conexión emocional entre el jugador y los ciudadanos y pone a estos bajo la constante presión de temperaturas menguantes y recursos escasos. Las difíciles condiciones hacen mella en el descontento y la esperanza de los ciudadanos, dos indicadores que de descontrolarse supondrán la expulsión del poder del jugador. Este deberá crear la estructura legal necesaria para la supervivencia, pero marcada por difíciles decisiones; entre ellas, hasta dónde llegar en los dos caminos que se le presentan: la instauración progresiva de una dictadura basada en el orden y la obediencia social, o de una teocracia. Se trabajará aquí con la versión básica de Frostpunk (11 bit studios, 2018) (sin expansiones) y su historia principal.

\section{Desarrollo metodológico: presentación de indicadores}

\subsection{Variables del sistema político}

Las siguientes variables reflejan la estructura política de la comunidad, así como la distribución de poder entre los actores.

Tabla 1. Variables 1, 2 y 3

\begin{tabular}{|l|l|r|r|r|}
\hline \multicolumn{1}{|c|}{ Variable } & \multicolumn{1}{|c|}{ Opciones } & Tropico & Civilization & Frostpunk \\
\hline $\begin{array}{l}\text { Competencias de } \\
\text { actuación de los } \\
\text { ciudadanos frente } \\
\text { al jugador }\end{array}$ & $\begin{array}{l}\text { 1. Nulas } \\
\text { 2. Bajas } \\
\text { 3. Medias } \\
\text { 4. Notables } \\
\text { 5. Altas }\end{array}$ & 2 & 5 \\
\hline $\begin{array}{l}\text { Nivel de } \\
\text { institucionalización } \\
\text { del conflicto político }\end{array}$ & $\begin{array}{l}\text { 1. Nula } \\
\text { 2. Media } \\
\text { 3. Alta }\end{array}$ & 2 & 1 & 1 \\
\hline $\begin{array}{l}\text { Igualdad social } \\
\text { y distribución } \\
\text { de recursos }\end{array}$ & $\begin{array}{l}\text { 1. Sin diferenciación social } \\
\text { 2. Diferenciación social con } \\
\text { igualdad material } \\
\text { 3. Diferenciación social con } \\
\text { desigualdad material }\end{array}$ & 3 & 2 & \\
\hline
\end{tabular}

Fuente: elaboración propia. 
En su revisión de estudios sobre calidad democrática, Tusell Collado sugiere que existen tres enfoques dominantes respecto a las características de una democracia: un enfoque procedimental, centrado en la selección de líderes políticos; un segundo que incorpora la relevancia del control político y, en último lugar, la idea de una democracia sustantiva que genere igualdad y bienestar social (Collado, 2015: 181-184).

La traslación de estos conceptos a los simuladores de gobierno es compleja. En primer lugar, la selección de líderes raramente es relevante, en tanto que la partida solo se produce en el escenario en el que el jugador es líder. Pero otros aspectos procedimentales pueden estar presentes, como la celebración de elecciones. Los simuladores de gobierno raramente presentan un conjunto de normas procedimentales democráticas detalladas, pero todo sistema de resolución de conflictos políticos institucionalizado se tiene en cuenta en la variable de «institucionalización del conflicto político». Esta variable se construye con tres opciones: en la nula institucionalización, los conflictos sociales no se resuelven a través de ningún cauce legal. En la institucionalización media, existen algunas herramientas legales que permiten el contrapoder legal y explícito, pero en absoluto existe un estado de derecho. En la institucionalización alta existe una democracia con instituciones desarrolladas y reglas en las que se gestiona el conflicto social.

Por otra parte, la idea de control político mantiene su importancia en los juegos, y se representa a través de la primera de estas variables, que empleando la terminología de Latorre (2010) llamamos «Competencias de actuación de los ciudadanos frente al jugador». A grandes rasgos, esta representa el «poder» que los ciudadanos pueden ejercer contra el jugador para exigir determinadas políticas o forzar su actuación en cierto ámbito, ya sea con métodos directos o indirectos (e incluso algunos que difícilmente podríamos considerar políticos). Algunos sub-indicadores de poder popular que pueden ayudar a establecer la puntuación de cada juego son los siguientes:

1. Los ciudadanos tienen voz, realizan peticiones $u$ opinan sobre la situación de la comunidad.

2. Responden a las políticas del gobernante con métodos indirectos que no representan oposición: abandonar la ciudad, luchar con menos fuerzas...

3. Se levantan en armas contra el jugador en casos extremos.

4. Bloquean la acción política del jugador.

5. Expulsan al jugador del poder por métodos formales (elecciones) o informales. 
Por último, el tercer enfoque, la idea de democracia sustantiva, puede representarse parcialmente a través de la variable de igualdad social, que incorpora tanto la existencia de desigualdades como de procesos de diferenciación social, normalmente basados en un funcionalismo laboral (los ciudadanos se dividen en trabajadores especializados y no especializados, por ejemplo). Cabe señalar que, al contrario que muchos estudios sobre democracia, este modelo no puede medir la amplitud de la desigualdad, pero sí podría mostrar una tendencia relevante en los simuladores de gobierno hacia el igualitarismo.

De ese modo, este modelo busca adaptarse a la realidad de los simuladores de gobierno, parte del conflicto social como problema sobre el que se construye la democracia y asume una posición que combina una idea sustantiva y material de la democracia, centrada en la distribución de la capacidad de influencia social (y no solo electoral) con una perspectiva institucional. Al mismo tiempo, se trata de un modelo que puede aplicarse a sistemas no democráticos (como posiblemente muchos simuladores de gobierno).

Por otra parte, al considerar a los ciudadanos como un único grupo, el modelo no tiene en cuenta la posibilidad de que la capacidad de influencia política sea distinta entre estos. En nuestro conocimiento de simuladores de gobierno, existen varios juegos donde los ciudadanos se dividen en grupos de interés (como las sagas Tropico o Democracy), pero tienen similar capacidad de influencia. Con todo, el modelo se encontraría con problemas en situaciones en las que no fuera así.

Tanto Tropico como Frostpunk han sido considerados «simuladores de dictadura», y con buenas razones para ello. Sin embargo, en nuestro modelo ofrecen niveles de democracia muy superiores a los de Civilization: en Frostpunk, el jugador se encuentra siempre bajo la presión de ser expulsado por sus ciudadanos si los indicadores de descontento y esperanza alcanzan sus límites, con lo que obtiene la máxima puntuación en competencias de actuación de los ciudadanos. Pero esa expulsión no está institucionalizada, es un levantamiento social espontáneo; se trata de un modelo sin instituciones de regulación del conflicto, un sistema con el germen de la democracia (un equilibrio material de poder) pero no su desarrollo. En Tropico los ciudadanos tienen más o menos el mismo poder, pero con la particularidad de que existen instituciones democráticas, aunque corruptas y manipulables por el jugador, que puede cometer fraude electoral o perseguir a la oposición. Esos actos, con todo, tendrán un coste para el jugador, con lo que Tropico obtiene una puntuación media en institucionalización del conflicto. 
Civilization, sin embargo, carece de cualquier institución para la gestión del conflicto, y el poder de los ciudadanos es bastante limitado. Cuando la infelicidad se sitúa entre 0 y menos 10 se producen efectos pasivos que difícilmente podríamos considerar como oposición: disminuye el crecimiento poblacional y los soldados luchan peor. Solo a partir de mayores niveles de infelicidad, algo extremadamente raro en el juego, aparecen grupos de rebeldes.

Al mismo tiempo, tanto Frostpunk como Civilization dividen a la sociedad entre especialistas y trabajadores ordinarios, pero sin que esto afecte a la distribución de recursos. Solo en Tropico los salarios y condiciones de vida pueden variar en función del lugar de trabajo y el nivel formativo, aunque las condiciones de desigualdad pueden aumentar o disminuir por causa de las políticas del jugador de una forma que el modelo no contempla.

Así pues, el simulador de dictaduras caribeñas Tropico aparece como el sistema político con mayor calidad de la democracia de entre los tres, en particular si los niveles de desigualdad se mantienen en un grado razonable. Eso no significa que su democracia sea ideal (todo lo contrario), pero parece relativamente garantista para la media de los simuladores de gobierno (que, por otra parte, podría medirse a partir de este modelo).

Tabla 2. Variable 4

\begin{tabular}{|l|l|r|r|r|}
\hline \multicolumn{1}{|c|}{ Variable } & \multicolumn{1}{|c|}{ Opciones } & Tropico & Civilization & Frostpunk \\
\hline $\begin{array}{l}\text { Competencias de } \\
\text { actuación del jugador: }\end{array}$ & 1. Baja & 2 & 3 & 3 \\
planificación económica & 2. Media & & & \\
\hline
\end{tabular}

Fuente: elaboración propia.

Este aspecto ha sido raramente tratado en los análisis sobre simuladores de gobierno, pero presenta interés en términos ideológicos. Asumiendo que el jugador encarna la figura del estado ${ }^{1}$, su capacidad de gestión de la economía es paralela al nivel de planificación económica del juego. Hemos establecido pues tres opciones: la planificación económica baja corresponde a un estado moderno liberal, en el que el jugador tiene acceso a políticas públicas que permiten incentivar una u otra actividad, pero la mayor parte de la actividad económica queda fuera de su control. En la planificación media, el jugador dispone de muy amplias herramientas de control y actividad, pero existe un amplio espacio para la iniciativa privada. Por último,

\footnotetext{
${ }^{1}$ Preferimos hablar de «estado» a «gobernante» porque los simuladores de gobierno suelen incorporar un fuerte componente de despersonalización y un recurso a informaciones estadísticas y capacidades de organización que hacen mucho más verosímil identificar la posición del jugador con el cuerpo abstracto del estado que con la de un simple gobernante.
} 
en la planificación alta, toda o prácticamente toda la actividad económica es dirigida directamente por el jugador.

En nuestra pequeña muestra, observamos que el nivel de planificación económica suele ser muy alto (en particular si lo comparamos con nuestros estados modernos), y solo es medio en Tropico, el juego de ambientación más moderna.

Tabla 3. Variable 5

\begin{tabular}{|c|c|c|c|c|}
\hline Variable & Opciones & Tropico & Civilization & Frostpunk \\
\hline $\begin{array}{l}\text { Unidad de la } \\
\text { comunidad } \\
\text { política } \\
\text { (representación) }\end{array}$ & $\begin{array}{l}\text { 1. La comunidad política } \\
\text { encuentra una unidad } \\
\text { completa, prácticamente } \\
\text { nunca se divide } \\
\text { 2. La comunidad política } \\
\text { encuentra momentos de } \\
\text { división, pero son raros } \\
\text { 3. La comunidad política } \\
\text { muestra habitualmente } \\
\text { división }\end{array}$ & 3 & 1 & 2 \\
\hline
\end{tabular}

Fuente: elaboración propia.

Esta cuestión ha sido considerada también en varios análisis sobre simuladores políticos. Planells (2015b) estudia la forma en la que Tropico presenta una sociedad dividida en grupos de interés que representan el pluralismo político de la sociedad, alineándose con distintas concepciones de democracia liberal (por ejemplo, la noción de poliarquía de Robert Dahl (2000). Por el contrario, Poblocki (2002) señala la metáfora del cuerpo humano y la multiplicación de las células para hablar de Civilization, mostrando una comunidad política inseparable. Esas metáforas biologicistas, y en muchos casos funcionalistas, han estado también presentes en las representaciones históricas de comunidades políticas: el Leviatán de Hobbes (1989), por ejemplo, se encontraba formado por los cuerpos de los ciudadanos. En Frostpunk, por último, se observan ciertos momentos de división, pero en general vuelve siempre a una reunificación social, ya sea en torno al líder, o incluso contra él (en el caso de su expulsión). En particular, la reunificación social que se produce después de un momento de división (el surgimiento de los Londinenses, que desean abandonar la comunidad) presenta toda la estructura del «estado de excepción» de Carl Schmidt (1998). 


\subsection{Variables de estructura de objetivos e incentivos a la acción}

Los siguientes indicadores se refieren a aquellos aspectos del juego que motivan o incentivan determinadas acciones para los jugadores. Este tipo de incentivos orientan a la comunidad política y establecen prioridades para sus recursos. En nuestras sociedades contemporáneas, esto es un asunto de debate público habitual (por ejemplo, con cuestiones como cuánto debemos invertir para frenar la emergencia climática, o reforzar los servicios públicos), por lo que su traslación a mundos videolúdicos conecta con esos debates.

Latorre (2010) emplea el término gameplay para describir las prácticas de juego esperadas y, en gran medida, diseñadas. Considera tres criterios para definir la (o las) gameplays de un juego:

a) Criterio de «eficacia» o del «jugador racional».

b) Criterio de coherencia discursiva.

c) Criterio del «lector modelo» (Latorre, 2010: 144).

El criterio de eficacia se refiere a la actuación con racionalidad de acuerdo a fines de los jugadores en pro de los objetivos del juego, e incorpora por tanto la búsqueda de estrategias más eficientes. El criterio de coherencia discursiva supone que, en particular en escenarios de alta inmersión, el jugador tiende a seguir la lógica narrativa y estética del juego. Por último, el criterio del «lector modelo» incorpora tanto las normas culturales en torno al juego como los aspectos estéticos del propio juego.

Dado que nuestro modelo se centra en la dimensión ludológica del juego, el criterio de eficacia es el que más consideramos, por encima de las dimensiones estéticas. Aunque esto facilite el análisis también lo hace menos completo.

Tabla 4. Variable 6

\begin{tabular}{|c|c|c|c|c|}
\hline Variable & Opciones & Tropico & Civilization & Frostpunk \\
\hline Objetivos de & Varias opciones pueden ser & $2(E)$ & $1(E)$ & 2 \\
\hline juego & marcadas & & 2 & $3(E)$ \\
\hline explícitos & 1. Dominación militar & $9(E)$ & 4 & 4 \\
\hline e implícitos & 2. Desarrollo económico & $10(E)$ & $5^{2}(E)$ & \\
\hline & o del nivel de vida & $11(\mathrm{E})$ & $6(\mathrm{E})$ & $9(E)$ \\
\hline & $\begin{array}{l}\text { 3. Supervivencia ante } \\
\text { condiciones naturales }\end{array}$ & & $7(\mathrm{E})$ & $11(\mathrm{E})$ \\
\hline & complejas & & $11(\mathrm{E})$ & \\
\hline & $\begin{array}{l}\text { 4. Crecimiento de } \\
\text { la comunidad política }\end{array}$ & & & \\
\hline
\end{tabular}

${ }^{2}$ Civilization $V$ cuenta con varios objetivos de victoria posibles, todos incluidos aquí. 


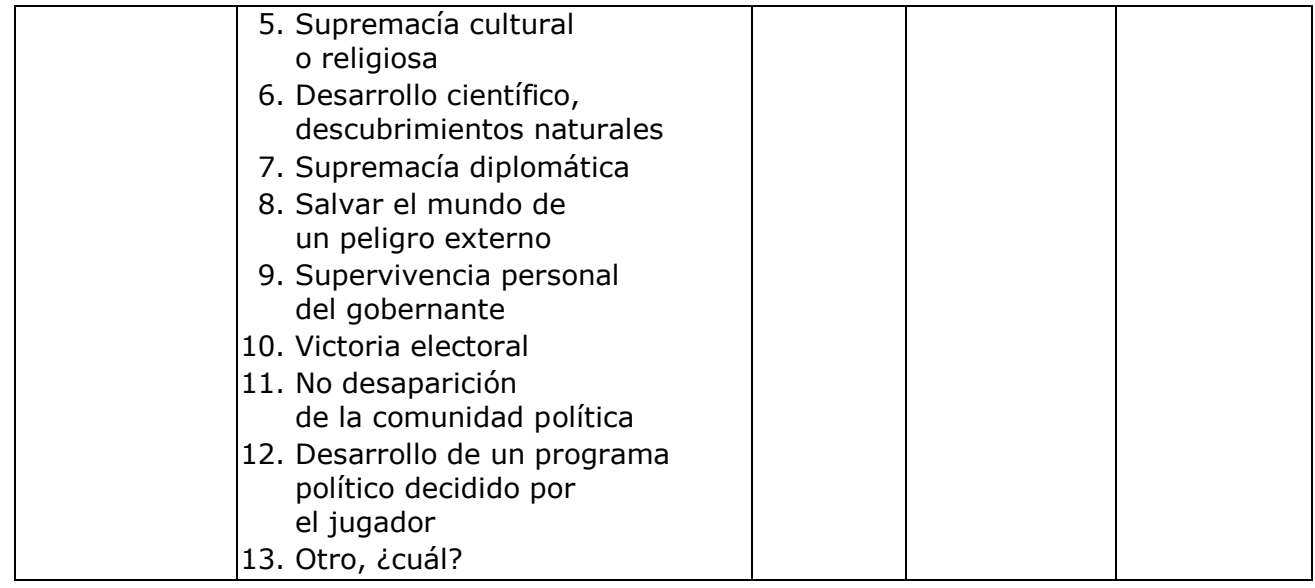

Fuente: elaboración propia.

La estructura de objetivos es uno de los aspectos comúnmente analizados en los estudios sobre videojuegos (Oliva et al., 2009). Al fin y al cabo, los objetivos no solo marcan la victoria o derrota, sino que orientan toda la acción del jugador, que actuará acorde a ellos.

Los objetivos explícitos definen la victoria o derrota formal en el juego, por lo que están algo limitados. Sin embargo, la categoría de objetivos implícitos incluye tanto aquellas estrategias prácticamente imprescindibles para lograr objetivos explícitos (lo cual no incluye estrategias óptimas pero prescindibles) como aquellos objetivos que el juego incentiva en los jugadores a través de componentes narrativos o estéticos. Al incorporar ambas nociones se busca superar levemente la limitación señalada anteriormente con respecto a los criterios para definir la gameplay. Una «(E)» señala que ese objetivo es explícito.

Muchos de los objetivos son compartidos, como el crecimiento de la comunidad política o el desarrollo económico y del nivel de vida: estos casos suelen ser objetivos implícitos, no establecidos como tal pero prácticamente imprescindibles para sobrevivir o ganar. Esto sugiere una presencia de mecánicas compartidas a pesar de que los objetivos explícitos sean distintos. En este caso, Frostpunk gira en torno a una lógica de supervivencia frente al desastre climático, Tropico de crecimiento y desarrollo económico, y Civilization de supremacía mundial, aunque con distintas posibilidades para lograrlas, pero los tres comparten, por ejemplo, una lógica desarrollista. 
Tabla 5. Variable 7

\begin{tabular}{|l|l|r|r|r|}
\hline \multicolumn{1}{|c|}{ Variable } & \multicolumn{1}{|c|}{ Opciones } & Tropico & Civilization & Frostpunk \\
\hline $\begin{array}{l}\text { Competencias de actuación } \\
\text { del jugador: dirección } \\
\text { política }\end{array}$ & $\begin{array}{l}\text { 1. Bajo } \\
\text { 2. Medio }\end{array}$ & 3 & 2 & 3 \\
3. Alto & & & \\
\hline
\end{tabular}

Fuente: elaboración propia.

La variable relacionada con la dirección política tiene que ver con el grado de libertad de los jugadores para desarrollar ideológicamente la comunidad política, un aspecto relevante para la capacidad del juego de estimular la imaginación política.

Dado que la variable presenta un grado importante de interpretación, hemos limitado a tres las opciones para facilitar la aplicación e identificar tendencias generales. En niveles de dirección altos, el jugador puede modificar su comunidad política y sus objetivos de forma fundamental, mientras que, en niveles de dirección bajos, gran parte del modelo organizativo y la definición ideológica de la comunidad se encuentran previamente marcados y sus cambios son imposibles o encuentran fuertes resistencias en los ciudadanos, con lo que el desarrollo es fundamentalmente técnico.

En este caso, Tropico y Frostpunk obtienen máximas puntuaciones en la escala porque permiten al jugador decretar leyes y tomar decisiones relevantes sobre su desarrollo (por ejemplo, escogiendo hasta qué punto desarrollar una agenda autoritaria en Frostpunk o priorizando a unas u otras facciones en Tropico). En Civilization encontramos menos momentos de decisión, y suelen presentarse de forma muy orientada a objetivos (a la hora de escoger la ideología importan mucho las bonificaciones de cada una con relación al tipo de victoria que se busca). Con todo, existe cierta libertad de elección (por ejemplo, por la pluralidad de objetivos de victoria), con lo que obtiene una puntuación intermedia.

Tabla 6. Variable 8

\begin{tabular}{|c|c|c|c|c|}
\hline Variable & Opciones & Tropico & Civilization & Frostpunk \\
\hline $\begin{array}{l}\text { Importancia } \\
\text { de la guerra } \\
\text { en la resolución de } \\
\text { conflictos externos }\end{array}$ & $\begin{array}{l}\text { 1. Inexistente } \\
\text { 2. Baja } \\
\text { 3. Media } \\
\text { 4. Alta } \\
\text { 5. No aplica (no existen } \\
\text { conflictos externos u } \\
\text { otras comunidades } \\
\text { políticas) }\end{array}$ & 1 & 3 & 5 \\
\hline
\end{tabular}

Fuente: elaboración propia. 
La potencial incitación de los videojuegos a la violencia ha sido un objeto de gran debate social y académico, aunque siempre sin resultados contundentes (Goldstein, 2005). Para los simuladores de gobierno, y considerando que los orígenes del género de estrategia (en el que se engloban) se encuentran en la estrategia militar (Dor, 2018), la predominancia de componentes bélicos en los juegos ha sido una preocupación habitual. Esta variable analiza la importancia de la guerra en la acción del jugador, y tiene en cuenta factores como la obligatoriedad o no para la consecución de los objetivos implícitos o su presencia general en la dinámica del juego.

En este caso, los juegos vuelven a tener características similares en función del género. La guerra es siempre un elemento en los $4 \mathrm{X}$ (una de las $\mathrm{X}$ significa «exterminate»), pero es bastante rara en los city builders. En el caso de Frostpunk no existen otras comunidades políticas, mientras que en Tropico es posible ser invadido por una de las superpotencias de la Guerra Fría, pero en ningún caso iniciar tú la escalada militar. En Civilization la guerra es obligatoria solo en una de las posibles victorias, pero es una estrategia relativamente ventajosa en muchos contextos, por lo que obtiene una puntuación intermedia en la escala.

Tabla 7. Variable 9

\begin{tabular}{|c|c|c|c|c|}
\hline Variable & Opciones & Tropico & Civilization & Frostpunk \\
\hline $\begin{array}{l}\text { Presencia de } \\
\text { límites } \\
\text { ecológicos } \\
\text { relevantes al } \\
\text { desarrollo }\end{array}$ & $\begin{array}{l}\text { 1. La actividad económica e } \\
\text { industrial provoca daños } \\
\text { relevantes en el ecosistema } \\
\text { o a la comunidad política } \\
\text { 2. La actividad económica o } \\
\text { industrial agota algunos } \\
\text { recursos, pero no es } \\
\text { perjudicial para el } \\
\text { ecosistema } \\
\text { 3. Todos o casi todos los } \\
\text { recursos son infinitos y el } \\
\text { desarrollo económico no } \\
\text { tiene consecuencias } \\
\text { medioambientales }\end{array}$ & 1 & 3 & 2 \\
\hline
\end{tabular}

Fuente: elaboración propia.

La problemática de una ideología de expansión sin límites ha sido señalada con respecto a varios simuladores de gobierno, como Civilization (Oliva et al., 2009) o Sim City (Atkins, 2003: 129), por sus consecuencias ecológicas. Esta variable es una forma de medir la dimensión ecológica desde esa perspectiva, pero no puede decirse que mida «lo ecologista» que es un juego, algo que requeriría un análisis en profundidad. En Frostpunk, por 
ejemplo, al ignorar los aspectos narrativos no se incorpora la dimensión ecologista que algunos autores identificaron por el desastre natural que causa la desaparición de la civilización antes del juego (Honeybun-Arnolda y Obermeister, 2019), sino que se le asigna un 2 porque unos pocos recursos son agotables. En Tropico la existencia de polución por actividades industriales y la presencia de una facción ecologista supone la asignación de un 3, lo contrario que en Civilization, donde excepto casos anecdóticos la contaminación no es un problema y los recursos son infinitos.

Tabla 8. Variables 10 y 11

\begin{tabular}{|l|l|r|r|r|}
\hline \multicolumn{1}{|c|}{ Variable } & \multicolumn{1}{|c|}{ Opciones } & Tropico & Civilization & Frostpunk \\
\hline $\begin{array}{l}\text { Existencia de } \\
\text { indicadores de } \\
\text { bienestar y } \\
\text { felicidad en la } \\
\text { población }\end{array}$ & $\begin{array}{l}\text { 1. No existen } \\
\text { 2. Existen, son poco } \\
\text { complejos } \\
\text { 3. Existen y son } \\
\text { complejos }\end{array}$ & 3 & 2 & 3 \\
\hline $\begin{array}{l}\text { Relevancia de los } \\
\text { indicadores de } \\
\text { bienestar y } \\
\text { felicidad en la } \\
\text { población }\end{array}$ & $\begin{array}{l}\text { 1. Nula } \\
\text { 2. Importancia localizada } \\
\text { 3. Importancia general }\end{array}$ & 3 & & 2 \\
\hline
\end{tabular}

Fuente: elaboración propia.

Más allá de los objetivos formales de cada juego, los simuladores políticos incorporan habitualmente una dimensión de «buen gobierno», sostenida sobre indicadores de bienestar o felicidad de la población. Esos indicadores suelen tener un impacto en las posibilidades del jugador de alcanzar la victoria; Shields, por ejemplo, afirma que en Tropico el jugador se ve obligado a seguir un cierto «contrato social» con los ciudadanos para evitar que se organicen en guerrillas y saboteen las infraestructuras de la isla (Shields, 2009). Dolkemeyer (2020), por su parte, señala que en Frostpunk existe una integración estética e inmersiva del jugador en la comunidad política, lo que le lleva a «vivir con y morir con» la comunidad, reforzando la experiencia emocional (algo que como jugador de Frostpunk puedo confirmar en gran medida). Estas dos dinámicas reflejan también consideraciones democráticas interesantes, tanto desde la perspectiva contractualista de Shields como desde una cierta idea de democracia con identificación entre gobernantes y gobernados en Frostpunk, donde el gobernante comparte parcialmente las experiencias vitales (o al menos algunas sensoriales) de los gobernados.

Estas variables no consideran tanto los aspectos estéticos referidos por Dolkemeyer, como los sistémicos de Shields. Sirven para analizar la forma en 
la que la satisfacción de los ciudadanos es representada, y su importancia a la hora de conseguir los objetivos formales. Encontramos un comportamiento similar de los juegos de construcción de ciudades frente al 4X. Tanto en Frostpunk como en Tropico hay indicadores detallados de bienestar y satisfacción que tienen en cuenta aspectos materiales e ideológicos y cuya mala gestión llevará al derrocamiento del jugador. En Civilization, sin embargo, existe un único indicador de felicidad de cuestionable modelización, y en general las consecuencias de mantenerlo por debajo de cero se limitan a un menor crecimiento poblacional.

\subsection{Variables de marcas enunciativas}

Tabla 9. Variables 12 y 13

\begin{tabular}{|c|c|c|c|c|}
\hline Variable & Opciones & Tropico & Civilization & Frostpunk \\
\hline $\begin{array}{l}\text { Representación } \\
\text { del avatar y el } \\
\text { punto de vista }\end{array}$ & $\begin{array}{l}\text { 1. No existe avatar, o solo } \\
\text { puede observarse en } \\
\text { imágenes o infiriéndolo a } \\
\text { partir de un punto de vista } \\
\text { en situaciones muy concretas } \\
\text { 2. El avatar está presente en la } \\
\text { partida, pero el punto de } \\
\text { vista no se corresponde con } \\
\text { él } \\
\text { 3. Existe un avatar y el punto } \\
\text { de vista se corresponde con } \\
\text { él }\end{array}$ & 2 & 1 & 1 \\
\hline $\begin{array}{l}\text { Construcción } \\
\text { estética de la } \\
\text { perspectiva } \\
\text { ética }\end{array}$ & $\begin{array}{l}\text { 1. Serious game con conexión } \\
\text { emocional } \\
\text { 2. Neutral-aséptico orientado a } \\
\text { objetivos } \\
\text { 3. Paródico-cínico }\end{array}$ & 3 & 2 & 1 \\
\hline
\end{tabular}

Fuente: elaboración propia.

La aparición de este grupo surge directamente del trabajo de Pérez Latorre (2010) y la incorporación de la semiótica y la teoría de la enunciación en sus análisis. El punto de vista ha sido considerado como un elemento que refuerza o disminuye la implicación del jugador en la trama, en función de su cercanía o alejamiento al del avatar. Sin embargo, en los simuladores de gobierno se da una habitual «desaparición del avatar» que, combinada con una ocularización «a vista de pájaro» y el recurso sistemático a paneles estadísticos, puede llevarnos a pensar en una encarnación del estado por parte del jugador. Por otra parte, la segunda variable, «construcción estética de la perspectiva ética», explora la forma en la que el diseño estético del juego incentiva, o no, relaciones empáticas o emocionales y exigencias éticas para 
los jugadores. Los tres juegos seleccionados representan tres tipos de enfoques diferentes: el cinismo de Tropico, que relativiza los componentes emocionales con un tono paródico, el realismo de Frostpunk, que busca construir una relación empática entre el jugador y sus ciudadanos a través de la inmersión y la representación de su sufrimiento (Dolkemeyer, 2020), y el modelo más técnico y orientado a objetivos de Civilization, donde las consecuencias de las acciones del jugador en las vidas o muertes de los ciudadanos solo se representan a través de indicadores genéricos (por ejemplo, si en Frostpunk la muerte de una persona es anunciada con campanas, mensajes informativos y visitas de los ciudadanos al cementerio, en Civilization la muerte de miles de ellos se verá únicamente reflejada en la disminución de un número representando la población de una ciudad).

\subsection{Variables independientes}

Tabla 10. Variables 14, 15, 16, 17 y 18

\begin{tabular}{|c|c|c|c|c|}
\hline Variable & Opciones & Tropico & Civilization & Frostpunk \\
\hline Género & $\begin{array}{l}\text { 1. Constructor de ciudades } \\
\text { 2. } 4 \mathrm{X} \\
\text { 3. Simulador político moderno } \\
\text { 4. Juego «de Dios» } \\
\text { 5. Otro }\end{array}$ & 1 & 2 & 1 \\
\hline Ambientación & $\begin{array}{l}\text { 1. Histórico realista } \\
\text { 2. Histórico fantástico } \\
\text { 3. Steampunk } \\
\text { 4. Era industrial } \\
\text { 5. Actualidad-siglo XX } \\
\text { 6. Ciencia ficción } \\
\text { 7. Varias etapas } \\
\text { 8. Otro }\end{array}$ & 5 & 1 & 3 \\
\hline Año (etapa) & $\begin{array}{l}\text { 1. } 1990-1999 \\
\text { 2. } 2000-2009 \\
\text { 3. } 2010-2015 \\
\text { 4. } 2016-2020 \\
\end{array}$ & 3 & 3 & 4 \\
\hline Año (concreto) & Respuesta abierta & 2011 & 2010 & 2018 \\
\hline $\begin{array}{l}\text { Tipo de } \\
\text { comunidad } \\
\text { política }\end{array}$ & $\begin{array}{l}\text { 1. Ciudad o asentamiento } \\
\text { estándar } \\
\text { 2. Ciudad o asentamiento de } \\
\text { refugiados } \\
\text { 3. Estado nación moderno } \\
\text { 4. Imperio } \\
\text { 5. Otro }\end{array}$ & 1 & 4 & 2 \\
\hline
\end{tabular}

Fuente: elaboración propia. 
Estas últimas variables son aquellas que consideraremos independientes, en tanto que pueden tener un efecto causal sobre las demás. Los efectos de algunas de estas variables ya fueron sugeridos por algunos autores. Por ejemplo, McNeil (2016) compara la quinta entrega de la saga Civilization con Beyond Earth (Firaxis Games, 2014), una entrega en paralelo que en lugar de ambientarse de forma histórica lo hace en un universo de ciencia ficción, en la colonización de un planeta desconocido. Destaca cómo esa nueva ambientación da pie a grandes diferencias ideológicas, en particular en relación a tres aspectos: el determinismo tecnológico (de la casi linealidad del árbol de tecnología se pasa a una mayor libertad de elección representada con un círculo), la representación del «otro» (los bárbaros son ahora alienígenas con los que el jugador puede mantener una buena relación) o el entorno (ahora más amenazante, desconocido y peligroso). Es razonable pensar que otras variables independientes, como el año de surgimiento del juego o el tipo de comunidad política, correlacionen de forma significativa con las variables dependientes, algo que sabríamos aplicando el modelo a gran escala e interpretando los resultados.

\section{Discusión de resultados: límites del modelo}

Varios problemas pueden ser señalados con relación al modelo y su aplicación:

- Las variables de escala tienen fronteras porosas: en las variables de escala, en ocasiones es complicado establecer si a un juego se le debe asignar un «4» o un «5». Aunque se han establecido sub-indicadores para ello, estos no siempre son exhaustivos, dada la gran pluralidad de realidades videolúdicas. Por ello, es común que nos encontremos con la sensación de que una escala no es lo suficientemente precisa, y que dos juegos presentan el mismo resultado a pesar de ser relativamente distintos. Este es, sin embargo, un límite aceptable y necesario a fin de lograr un modelo sencillo y aplicable.

- Las variables no deben confundirse con la realidad que buscan representar. Las variables son operacionalizaciones de realidades que queremos representar (como la democracia o el ecologismo), pero no sustituyen esa realidad, sino que representan una fracción reducida de ella. Tanto su aplicación como su configuración presentan además un cierto grado de arbitrariedad, a pesar de los esfuerzos porque sean rigurosas. Con todo, realizar juicios generales a partir de indicadores parciales es precisamente el objetivo de la operacionalización, y la prueba de la calidad de dichos indicadores. Recomendamos entonces una mirada crítica hacia el proceso de operacionalización, que tome el 
modelo como una herramienta de análisis antes que una representación de la realidad. Al mismo tiempo, en ocasiones algunos aspectos de un videojuego son excesivamente complejos para representarse de forma satisfactoria con pocas variables numéricas, por lo que pueden emplearse fichas cualitativas para complementar el análisis.

- El modelo no considera los cambios en el sistema político de un juego. En ocasiones, hay variables que sufren cambios durante la partida. Por ejemplo, las competencias de actuación de los ciudadanos al inicio de Frostpunk son altas, pero si el jugador alcanza los máximos grados de establecimiento de una dictadura totalitaria, estas se reducen ampliamente, llegando incluso a remover algunos de los indicadores que producen una revuelta. En este caso hemos tomado la situación inicial (al ser la obligatoria) como referencia, pero el modelo solo aporta una foto fija, estática, del sistema político en el que nos movemos. En un futuro podrían incorporarse al modelo variables destinadas a registrar su dinamismo.

- El modelo no tiene en cuenta los aspectos narrativos de los videojuegos. Este modelo está pensado para analizar el funcionamiento sistémico, ludológico, de los simuladores de gobierno. Podríamos establecer variables relacionadas con las posibles narrativas de cada juego, pero haría el modelo mucho más complejo y difícil de aplicar. Por ello hemos decidido evitar esa clase de análisis, aun sabiendo que ello implica una pérdida de información importante. Consideramos, sin embargo, que se podrían construir modelos comparativos de narrativas similares al aquí presentado para los aspectos sistémicos.

Sin duda, muchos más límites y problemas podrían mencionarse, pero esta breve selección puede ser un útil «aviso a navegantes» que quieran aplicar el modelo.

\section{Conclusiones}

A pesar de la creciente atención académica que los videojuegos reciben, el campo de los simuladores de gobierno y la forma en la que encarnan las ciencias políticas y de la administración requiere aún de investigaciones y discusiones en profundidad. Múltiples trabajos han señalado las conexiones en videojuegos concretos, pero el modelo que presentamos aquí pretende servir para dar una visión más general. Deberá ser discutido, ampliado y aplicado a muestras grandes de videojuegos para poder evaluarse de forma adecuada, y probablemente requiera de una combinación con métodos de análisis cualitativos para alcanzar su máxima utilidad. Con todo, esta primera 
aproximación puede ser útil tanto para señalar las oportunidades de investigación como para aportar algunas modestas herramientas para su análisis.

Pero más allá de los análisis de contenido, debemos entender mejor la forma en la que los jugadores interactúan con estos juegos y sus efectos en la socialización política. En un contexto social de resurgimiento de posiciones políticas autoritarias, los videojuegos, tradicionalmente criticados por su contenido político, podrían también convertirse en una oportunidad para mostrar la complejidad de la democracia y reflexionar en común sobre los asuntos públicos, involucrando en el proceso a académicos, diseñadores y jugadores en general. Las ficciones son fundamentales en una democracia porque tienen capacidad de estimular la imaginación política de sus ciudadanos, ofreciendo nuevos mundos posibles a los que tal vez queramos aspirar. Y en ese sentido, los juegos son fábricas de realidad y, los simuladores de gobierno, espacios de debate y experimentación.

\section{Referencias bibliográficas}

ATKINS, B. (2003). More than a game: The computer game as fictional form. Manchester University Press.

https://doi.org/10.7228/manchester/9780719063640.001.0001

Bogost, I. (2007). Persuasive Games: The Expressive Power of Videogames. MIT Press. https://doi.org/10.7551/mitpress/5334.001.0001

BURKE, K. (1945). A Grammar of Motives. Prentice-Hall.

ChAuRIYE, G. (2019). Frostpunk: decisiones con sentido para juegos con sentido. Ludology: revista de investigación sobre juegos y videojuegos, 1(1), 16-22.

DAHL, R. (2000). On Democracy. Yale University Press.

DolKEMEYER, L. (2020). Autocracy for the People. Modes of response-able Action and the Management of Demise in Frostpunk. Gamevironments, 13. Disponible en: https: //tinyurl.com/8a7fe8nf

DoR, S. (2018). Strategy in Games or Strategy Games: Dictionary and Encyclopaedic Definitions for Game Studies. Game Studies, 18(1). Disponible en: https: //tinyurl.com/cvjendm9

Douglas, C. (2002). «You Have Unleashed a Horde of Barbarians!»: Fighting Indians, Playing Games, Forming Disciplines. Postmodern culture, 13(1). https://doi.org/10.1353/pmc.2002.0029

Exmeyer, P. \& Boden, D. (2020). The 8-Bit Bureaucrat: Can Video Games Teach Us About Administrative Ethics? Public Integrity, 22(5), 1-16. https: //doi.org/10.1080/10999922.2020.1731056 
FORD, D. (2016). «eXplore, eXpand, eXploit, eXterminate»: Affective Writing of Postcolonial History and Education in Civilization V. Game Studies, 16(2).

GHYS, T. (2002). Technology Trees: Freedom and Determinism in Historical Strategy Games. Game Studies, (12).

Goldstein, J. (2005). Violent Video Games. En Raessens, J. \& Goldstein, J. (Eds.), Handbook of Computer Games Studies (pp. 341-359). MIT Press.

HoBBEs, T. (1989). Leviatán: la materia, forma y poder de un Estado eclesiástico y civil. Alianza.

HonEYBUn-ARNOLDA, E. \& OBERMEISTER, N. (2019). A climate for change: Millennials, science and the humanities. Environmental Communication, 13(1), 1-8. https: //doi.org/10.1080/17524032.2018.1500927

HUNICKE, R., LEBLANC, M. \& ZUBEK, R. (2004, Julio). MDA: A formal approach to game design and game research. In Proceedings of the AAAI Workshop on Challenges in Game AI. 4(1), 17-22.

MCNEIL, E. (2016). Ludic Spolia in Sid Meier's Civilization: Beyond Earth. Journal of games criticism, (3).

NAVARRO REMESAL, V. (2016). Libertad dirigida: una gramática del análisis y diseño de videojuegos. Shangrila.

OLIVA, M., BESALÚ, R. y CiAURRIZ, F. (2009). Más grande, más rápido, mejor: la representación de la Historia universal en Civilization IV. Comunicación, 7 (1), 62-79.

PÉREZ LATORRE, O. (2010). Análisis de la significación del videojuego. Fundamentos teóricos del juego, el mundo narrativo y la enunciación interactiva como perspectivas de estudio del discurso [Tesis Doctoral]. Universitat Pompeu Fabra, Barcelona.

PÉREZ LATORRE, O., OLIVA, M. y BESALÚ, R. (2016). Videogame analysis: a socialsemiotic approach. Social Semiotics, 27(5), 586-603.

https://doi.org/10.1080/10350330.2016.1191146

PÉREZ LATORRE, O., Oliva, M., CiaURRIZ, F. y GuERRERO-SolĖ, F. (2008). Jugar a científics: videojocs i divulgació científica. Quaderns del CAC, 30, 27-36.

PlANELLS, A. (2015a). Mundos posibles, grupos de presión y opinión pública en el videojuego Tropico 4. Trípodos, 37, 167-181.

Planells, A. (2015b). Videojuegos y mundos de ficción: de "Super Mario" a "Portal". Cátedra.

PoвŁOCKI, K. (2002). Becoming-state. Bio-cultural Imperialism of Sid Meier's Civilization. European Journal of Anthropology, 39, 163-177

SCHMIDT, C. (1998). Teología Política: cuatro ensayos sobre la soberanía. Struhart \& CIA. 
ShIElDS, M. (2009). A Pentadic Analysis of Tropico: Dramatism and Digital Games [Tesis para el Master of Arts in Interdisciplinary Studies, Oregon State University]. Disponible en: https://tinyurl.com/exvvjd6n

TuSEll Collado, A. (2015). La calidad de la democracia y sus factores determinantes. Un análisis comparado de 60 países. Política y Sociedad, 52(1), 179-204.

ZAmaróCZY, N. (2017). Are We What We Play? Global Politics in Historical Strategy Computer Games. International Studies Perspectives, 18(2), 155174. https: //doi.org/10.1093/isp/ekv010

\section{Ludografía}

Democracy 3, 2013. [Videojuego] (PC) Positech Games, Positech Games. Frostpunk, 2018. [Videojuego] (PC) 11 bit studios, 11 bit studios.

Sid Meier's Civilization: Beyond Earth, 2014. [Videojuego] (PC) Firaxis Games, 2K Games.

Sid Meier's Civilization V, 2010. [Videojuego] (PC) Firaxis Games, 2K Games. Sim City, 1989. [Videojuego] (PC). Maxis, Maxis.

Tropico IV, 2011. [Videojuego] (PC) Haemimont Games, Kalypso Media. 